\title{
Response to Dr. Russell Kirby
}

\author{
Heather Bradford
}

Published online: 7 May 2009

(C) Springer Science+Business Media, LLC 2009

This letter suggests that the premise of the study may be flawed by a misunderstanding of the process of administrative record documentation of birth events occurring in hospitals. On the contrary, we believe we are in concurrence with this author in understanding how administrative record documentation is done in hospital settings, and therefore must argue that variations in CNM and MD accuracy do exist. As clinicians, we can verify that we do not complete the medical portion of the birth certificate in consultation with the patient chart, but instead rely on this function to be performed by medical record staff or unit clerk, and believe this is the more common practice in WA State.

We agree that the most direct solution to this problem would be the development and implementation of

administrative databases that acquire data directly from clinical documentation systems. However, since this is not the case, we do find our research findings valuable and should be approached with more than a footnote. Our findings highlight the degree of inaccuracy in both birth attendant types, and second, for the first time, allows a comparison between the two, with several variables being statistically significant in the birth certificate and combined data sources. Given these inaccuracies, and the recording variations between birth attendants, it is important to be aware of the level of measurement error, particularly when using birth certificate data alone.
H. Bradford $(\square)$

Adjunct Clinical Faculty, University of Washington School of Nursing, 527 Kirkland Avenue, Kirkland, WA 98033, USA

e-mail: hbradford@comcast.net 